\title{
A hanseníase na atenção primária à saúde: Atividades educativas em saúde e seu papel preventivo
}

\author{
Leprosy in primary health care: Educational activities in health and its preventive role \\ Lepra en la atención primaria de salud: Actividades educativas en salud y su función preventiva
}

Recebido: 02/05/2021 | Revisado: 11/05/2021 | Aceito: 07/06/2021 | Publicado: 11/06/2021

Adriane Queiroz Gomes

ORCID: https://orcid.org/0000-0003-2408-124X

Universidade da Amazônia, Brasil

E-mail: adrianequeiroz123@outlook.com

Anna Carolina Rocha de Paiva

ORCID: https://orcid.org/0000-0001-6831-8718 Universidade do Estado do Pará, Brasil

E-mail: godoikawa4@gmail.com

Carina Costa Cardoso

ORCID: https://orcid.org/0000-0002-2083-9917

Universidade Federal do Pará, Brasil

E-mail: carinaccardoso.med@gmail.com

Clívia Moura Azevedo

ORCID: https://orcid.org/0000-0002-0250-1605

Universidade Federal do Pará, Brasil

E-mail: cli.azevedo@hotmail.com

Deborah Favacho dos Santos Baia

ORCID: https://orcid.org/0000-0001-9560-3370

Universidade Federal do Pará, Brasil

E-mail: favachodeborah@gmail.com

Iasmim Ianne Sousa Tavares

ORCID: https://orcid.org/0000-0003-2350-874X

Faculdade de Ensino Superior da Amazônia Reunida, Brasil

E-mail: iasmim.tavares14@gmail.com

Isabelle Joubert Pereira

ORCID: https://orcid.org/0000-0003-1006-2718

Universidade da Amazônia, Brasil

E-mail: isabelle.joubert@outlook.com

Jéssica Maria Lins da Silva

ORCID: https://orcid.org/0000-0003-3218-6447

Universidade do Estado do Pará, Brasil E-mail: jeeh.sylva@gmail.com

Kelem Bianca Costa Barros

ORCID: https://orcid.org/0000-0002-1161-6228

Universidade Federal do Pará, Brasil

E-mail: kelembianca@icloud.com

Liandra Silva Lopes

ORCID: https://orcid.org/0000-0001-5108-3967

Universidade do Estado do Pará, Brasil

E-mail: liaa.loopes@gmail.com

Maria Rosilda Valente de Sarges

ORCID: https://orcid.org/0000-0001-9689-718X

Universidade da Amazônia, Brasil

E-mail: mrvsfarma@gmail.com

Michele de Pinho Barreiros

ORCID: https://orcid.org/0000-0002-7444-6238

Universidade Estadual da Paraíba, Brasil

E-mail: mestrandamichele@gmail.com

Monike Karina Macedo Soares

ORCID: https://orcid.org/0000-0002-3349-5463

Universidade do Estado do Pará, Brasil

E-mail: monikemacedo2@gmail.com

Pedro Lucas Carrera da Silva

ORCID: https://orcid.org/0000-0002-7728-8554

Universidade do Estado do Pará, Brasil

E-mail: pedrolucas02890@gmail.com 


\author{
Tamires Costa Franco \\ ORCID: https://orcid.org/0000-0002-8978-4176 \\ Universidade da Amazônia, Brasil \\ E-mail: tamiresfranco98@gmail.com \\ Thiago Simplício Costa \\ ORCID: https://orcid.org/0000-0002-6349-7013 \\ Universidade do Estado do Pará, Brasil \\ E-mail: thiagosimplicio221@gmail.com
}

\begin{abstract}
Resumo
A hanseníase é uma doença infecciosa crônica causada pelo bacilo Mycobacterium leprae, com caráter altamente transmissível e relevante dentro do cenário de saúde pública brasileira. Estudos apontam que na região norte ocorreu um aumento significativo de casos detectados já em estágio avançado da doença, com alto grau de incapacidade funcional, o que enfatiza a prevalência de um diagnóstico tardio nesta localidade, bem como de fatores que influenciam diretamente no reconhecimento e tratamento prévio da doença, como os aspectos socioeconômicos e o desconhecimento acerca da doença. Assim, este estudo objetivou fomentar o reconhecimento dos principais aspectos que envolvem a hanseníase, sua mitigação e autonomia dos usuários mediante as principais medidas preventivas desta patologia. O presente estudo tem uma abordagem qualitativa e descritiva, sendo um relato de experiência acerca da vivência interdisciplinar de acadêmicos da saúde durante ações educativas, baseadas no Arco de Maguerez, em uma unidade de saúde de Belém-PA. Como resultado, muitos usuários da unidade estavam retraídos no início das ações, entretanto, percebeu-se maior entusiasmo após incentivos. Outrossim, durante conversas com a população no local, ficou claro o pouco conhecimento da doença, o que corrobora sua importância dentro dos serviços de saúde. Ademais, o público-alvo reiterou a importância da atividade para a prevenção dos casos graves da doença e convidou a equipe de acadêmicos para retornar com a realização de outras ações educativas. Identificou-se a importância da profilaxia da hanseníase no ambiente da atenção básica, além da atuação do acadêmico ao adquirir competências para futura profissão, pautada no olhar holístico.
\end{abstract}

Palavras-chave: Hanseníase; Prevenção; Educação em saúde.

\begin{abstract}
Leprosy is a chronic infectious disease caused by the bacillus Mycobacterium leprae, with a highly transmissible and relevant character within the Brazilian public health scenario. Studies point out that in the northern region there was a significant increase in cases detected at an advanced stage of the disease, with a high degree of functional disability, which emphasizes the prevalence of a late diagnosis in this location, as well as factors that directly influence the recognition and treatment disease, such as socioeconomic aspects and lack of knowledge about the disease. Thus, this study aimed to promote the recognition of the main aspects involving leprosy, its mitigation and users' autonomy through the main preventive measures of this pathology. The present study has a qualitative and descriptive approach, being an experience report about the interdisciplinary experience of health students during educational activities, based on the Arco de Maguerez, in a health unit in Belém-PA. As a result, many users of the unit were withdrawn at the beginning of the actions, however, there was greater enthusiasm after incentives. Furthermore, during conversations with the local population, it was clear that little knowledge of the disease was found, which corroborates its importance within health services. In addition, the target audience reiterated the importance of the activity for the prevention of severe cases of the disease and invited the academic team to return with other educational activities. The importance of leprosy prophylaxis in the primary care environment was identified, in addition to the role of the academic when acquiring skills for a future profession, based on a holistic view.
\end{abstract}

Keywords: Hansen's disease; Prevention; Health education.

\title{
Resumen
}

La lepra es una enfermedad infecciosa crónica causada por el bacilo Mycobacterium leprae, con un carácter altamente transmisible y relevante en el escenario de la salud pública brasileña. Estudios señalan que en la región norte hubo un aumento significativo de casos detectados en un estadio avanzado de la enfermedad, con un alto grado de discapacidad funcional, lo que enfatiza la prevalencia de un diagnóstico tardío en esta ubicación, así como factores que directamente influyen en el reconocimiento y tratamiento de la enfermedad, como los aspectos socioeconómicos y el desconocimiento de la enfermedad. Así, este estudio tuvo como objetivo promover el reconocimiento de los principales aspectos relacionados con la lepra, su mitigación y la autonomía de los usuarios a través de las principales medidas preventivas de esta patología. El presente estudio tiene un enfoque cualitativo y descriptivo, siendo un relato de experiencia sobre la experiencia interdisciplinaria de estudiantes de salud durante acciones educativas, con base en el Arco de Maguerez, en una unidad de salud en Belém-PA. Como resultado, muchos usuarios de la unidad se retiraron al inicio de las acciones, sin embargo, hubo mayor entusiasmo luego de los incentivos. Además, durante las conversaciones con la población local, quedó claro que se encontró poco conocimiento de la enfermedad, lo que corrobora su importancia dentro de los servicios de salud. Además, el público objetivo reiteró la importancia de la actividad para la prevención de casos severos de la enfermedad e invitó al equipo académico a regresar con otras actividades educativas. Se identificó la importancia de la profilaxis contra la lepra en el ámbito de la atención 
primaria, además del rol del académico a la hora de adquirir competencias para una futura profesión, con base en una visión holística.

Palabras clave: Enfermedad de Hansen; Prevención; Educación para la salud.

\section{Introdução}

A hanseníase é uma doença infecciosa e crônica causada pelo bacilo Mycobacterium leprae, com caráter altamente transmissível e relevante dentro do cenário de saúde pública brasileira, uma vez que suas implicações podem gerar graves lesões neurais. No Brasil, a patologia destaca-se por seu caráter endêmico, sendo o segundo país em número de casos no mundo, enfatizando sua fundamentalidade dentro da rede de saúde (Sousa, Silva, \& Xavier, 2017).

Além disso, apenas na região norte, onde este estudo foi vivenciado, ocorreu um aumento significativo de casos detectados já em estágio avançado da doença, com alto grau de incapacidade funcional, o que enfatiza a prevalência de um diagnóstico tardio nesta localidade, bem como de fatores que influenciam diretamente no reconhecimento e tratamento prévio da doença, como os aspectos socioeconômicos e o desconhecimento acerca da patologia (Ribeiro, Silva, \& Oliveira, 2018; Silvestre \& Lima, 2016).

Dentre os principais sinais e sintomas da doença destaca-se o acometimento da pele e nervos periféricos com perda gradual de sensibilidade, sendo facilmente diagnosticável e tratável dentro da atenção primária da saúde, dividindo-se em quatro formas clínicas: indeterminada, tuberculóide, virchowiana e dimorfa. Entretanto, identifica-se que o número de pessoas que não reconhecem os sintomas, ou ainda não têm ciência da disponibilidade do tratamento e de sua gratuidade, ainda são alguns dos motivos para a manutenção das taxas de incidência no país (Nunes, Oliveira, \& Vieira, 2011).

Além disso, destaca-se a importância do diagnóstico precoce e da interrupção da cadeia de transmissão para o combate efetivo da doença. Dessa forma, enfatiza-se o papel da atenção básica para o fomento das ações profiláticas, uma vez que esse nível caracteriza-se por empoderar o usuário acerca dos seus conhecimentos em saúde, estimulando a efetivação dos seus direitos e deveres em saúde, bem como oportunizando a utilização dos diversos serviços ofertados (Araújo, Dias, \& Bustorff, 2011).

No que concerne aos profissionais que estão envolvidos diretamente nos cuidados aos indivíduos com essa doença, destaca-se a figura da equipe multiprofissional da atenção básica, uma vez que eles podem contribuir ativamente para o cuidado, através da execução de uma assistência integral e holística, pautada nas necessidades e nas demandas específicas de cada indivíduo, atuando diretamente na humanização e sensibilização quanto à importância da adesão ao tratamento (Sousa $e t$ al., 2017).

Outrossim, salienta-se a figura do acadêmico atuante nos níveis assistenciais, uma vez que essa inserção pode promover maior autonomia dentro de seu desenvolvimento pessoal, bem como fomentar maior contato com diversas realidades fora dos muros da academia, contribuindo, assim, para o desenvolvimento de um olhar humanizado e equânime na assistência em saúde (Amaral, Pontes, \& Silva, 2014).

Desse modo, desenvolveu-se este estudo com base em uma vivência de acadêmicos de cursos da área da saúde, ocorrida em uma unidade básica, a partir da efetivação de uma ação de promoção à saúde, que teve como principal objetivo o fomento ao reconhecimento dos principais aspectos que envolvem a hanseníase, bem como sua gradual mitigação e empoderamento dos usuários da atenção básica através das principais medidas preventivas desta patologia.

\section{Metodologia}

Este artigo configura-se como um estudo de caráter descritivo com abordagem qualitativa, na forma de relato de experiência, o qual relata a prática interdisciplinar de acadêmicos de saúde em uma unidade de saúde localizada no centro da 
capital paraense em Belém-Pará.

A experiência e observação dos acadêmicos ocorreu na segunda semana do mês de agosto, de segunda à quarta, durante as atividades práticas do componente curricular obrigatório "Doenças Infecciosas e Parasitárias", cujo objetivo é levar aos discentes o conhecimento clínico e epidemiológico das principais doenças infecciosas e parasitárias do Brasil e da região na qual convivem, bem como reconhecer quadros clínicos, diagnósticos e tratamentos, além de orientar acerca do controle e profilaxia dessas doenças.

Durante as atividades, os discentes fizeram a observação em toda a unidade de saúde, assim assistiram as como consultas realizadas dentro do programa de hanseníase e conversas com usuários que buscavam atendimento para hanseníase na referida unidade de saúde.

Dessa maneira, optou-se por desenvolver o presente estudo com base na Teoria da Problematização, mediante as etapas do Arco de Maguerez, cujas etapas subsidiam o processo metodológico da identificação de problemáticas existentes dentro de determinadas situações e cenários, assim como na escolha de medidas de intervenção a serem efetuadas para a mudança de uma realidade observada. O Arco de Maguerez divide-se em cinco etapas: observação das condições da situação vivenciada, levantamento dos pontos-chave pelos observadores, teorização, levantamento das hipóteses de solução da problemática e retorno à situação para a devida aplicação da ação planejada (Berbel, 2011).

$\mathrm{Na}$ primeira fase do arco de Maguerez, foi possível observar as situações-problemas presentes na unidade, sendo levantado os pontos-chave da necessidade do trabalho com a temática hanseníase, haja vista a quantidade de usuários atendidos na unidade com sintomas da doença. Desse modo, com essas etapas concluídas, os discentes buscaram na literatura científica artigos que pudessem embasar a ação e fornecer maior repertório e conhecimentos acerca da doença. Posteriormente, desenvolveu-se a hipótese de solução a partir da escolha da ação educativa e, por fim, ocorreu o retorno ao local para sua aplicação.

A ação dividiu-se nas seguintes etapas: acolhimento inicial, seguido de uma roda de conversa com o uso de tecnologias leves para o debate acerca da hanseníase e, por fim, uma dinâmica para o esclarecimento e fixação das informações passadas. Ademais, foi repassado um folder para efetuar a disseminação do conteúdo, que contava com as informações passadas durante a ação. Participaram da atividade educativa 18 usuários da unidade de saúde, sendo que alguns já realizavam tratamento para a hanseníase, conjuntamente com os acadêmicos, a docente preceptora da prática em questão, o diretor da unidade e os profissionais de saúde do local, os quais contribuíram para a criação de vínculos dos discentes com os usuários e uma participação mais ativa deles.

\section{Resultados}

Como principais resultados obtidos durante a visita prévia para observação da realidade local, evidenciou-se que, nas consultas, grande parte dos usuários que buscavam atendimento para a sintomatologia da hanseníase apresentavam-se em estágio avançado da doença, fato que reitera as lacunas dentro da educação em saúde para o reconhecimento prévio da patologia. Além disso, durante conversas informais com a população adscrita no local, ficou claro o pouco conhecimento acerca da doença, o que contribui para sua continuidade dentro dos serviços de saúde.

A partir disso, durante a aplicação das etapas do arco de Maguerez, optou-se por desenvolver uma ação educativa com a população do local, através do uso de tecnologias leves, visando o empoderamento do usuário do sistema de saúde, através da atividade educativa acerca da doença e a importância de seu reconhecimento prévio. Assim, a ação de educação em saúde ocorreu nas seguintes etapas: roda de conversa sobre a doença e os aspectos principais que a envolvem; dinâmica para fixação das informações repassadas; e, por fim, distribuição de material impresso (folders) para repasse e disseminação do conteúdo. 
A ação ocorreu em um espaço ao ar livre cedido pelo gestor da unidade de saúde, contando com a presença de duas profissionais do local, os acadêmicos que implementaram a ação e dezoito usuários do serviço, que foram previamente convidados pelos profissionais do local para participar da ação. Inicialmente observou-se que a maioria dos usuários encontravam-se tímidos e retraídos, entretanto, à medida que os profissionais do local os incentivavam a participar, verificouse maior entusiasmo para a ação.

A equipe de acadêmicos abordou primeiramente os aspectos gerais da hanseníase, mostrando o agente etiológico da doença, os meios de transmissão, sintomatologia, formas de diagnóstico e tratamento, e as principais medidas para prevenir a doença. Assim, para facilitar essa discussão, utilizou-se um cartaz com ilustrações sobre as informações supracitadas, visando maior dinamização da atividade educativa.

Nessa etapa, evidenciou-se que os participantes estavam prestando atenção no que estava sendo repassado, assim como indagando a equipe quando surgiam dúvidas, as quais eram prontamente clarificadas pelos estudantes. Além disso, quatro participantes haviam tido caso de hanseníase na família e, durante a roda de conversa, relataram a importância do tratamento prévio para evitar maiores complicações da doença.

Posteriormente, ocorreu a dinâmica para fixação do que foi repassado, com um jogo de perguntas e respostas acerca da doença, sendo elas: "O que é a hanseníase?", "Como se transmite a doença?", "Quais os principais sinais e sintomas da doença?", "Quais as medidas de prevenção da hanseníase?”, “Como é realizado o diagnóstico da hanseníase?”, “Qual a importância do tratamento prévio?" e "Quais as reações comuns ao tratamento?".

As perguntas foram distribuídas aleatoriamente e à medida que os participantes escolhiam a sua, eles tentavam responder com base no que foi informado durante a roda de conversa. Nos casos em que houve dificuldade para responder, outros participantes eram chamados para complementar a resposta. Evidenciou-se durante esse momento que os usuários participaram ativamente, tentando responder corretamente a todas as perguntas. Além disso, percebeu-se a promoção da comunicação interpessoal saudável entre os participantes, que ajudavam uns aos outros a cumprir a demanda proposta. Assim, a cada resposta dada, o grupo de discentes fornecia um feedback para o público, ratificando ou retificando o que havia sido falado.

Por fim, ocorreu a distribuição de folders com as informações que haviam sido trabalhadas na ação educativa, visando sua disseminação, bem como fixação dos conhecimentos repassados. Ademais, o público-alvo reiterou a importância da atividade para a prevenção dos casos graves da doença, assim como, juntamente com a equipe de profissionais do local, convidou a equipe de acadêmicos para retornar com a realização de outras ações educativas para a comunidade.

\section{Discussão}

A hanseníase ainda é um problema importante de saúde coletiva devido a magnitude de pessoas que atinge, bem como seu alto poder incapacitante. Na comparação epidemiológica com os anos de 2017 e 2018 o Brasil está entre os três países, junto com Índia e Indonésia, que tiveram alta na incidência de casos, de modo que encontra-se na segunda posição mundial na incidência da doença, fator que se relaciona diretamente com as desigualdades socioeconômicas e regionais do país (Leite et al., 2020; Meneses et al., 2020; Rodrigues, Arcêncio, \& Lana, 2021).

O Brasil está perto de atingir a faixa de controle da Organização Mundial de Saúde (OMS), contudo cerca de 535 municípios ainda classificam-se como hiperendêmicos e com taxa de deteç̧ão de casos acima de 40/100.000 pessoas habitantes. A distribuição geográfica da hanseníase tem caráter desigual e persiste em regiões com maiores níveis de pobreza, com volumosos índices de moradias ocupadas e precárias situações de saúde e de vida. Nas regiões Norte, Nordeste e CentroOeste, com exceção do Rio Grande do Norte e Distrito Federal, ainda há presença de elevados coeficientes, cerca de uma detecção média de 59,19/100.000 habitantes, com destaque para estados do Mato Grosso, Tocantins, Rondônia, Maranhão e 
Pará (Rodrigues, Arcêncio, \& Lana, 2021; Silva, 2020; Silva \& Sousa, 2021).

Em uma análise de indicadores entre os anos de 2012 e 2016 a região Norte obteve uma das maiores taxas médias de detecção geral, 34,26/100.000 habitantes. Nos últimos anos a hanseníase vem sendo manejada apenas para o seu controle, ao invés de serem criadas ações para a eliminação da patologia. Assim, evidencia-se a integração de ações de controle no país, que passaram a ser a principal estratégia para se mitigar níveis endêmicos da hanseníase no Brasil (Bordon et al., 2019).

Dessa forma, considera-se que implementar ações no âmbito da Atenção Primária em Saúde (APS) seja a melhor estratégia no controle da hanseníase, visto que facilita o conhecimento de sinais e sintomas para a comunidade, assim como facilita o acesso ao diagnóstico oportuno, tratamento e a cura, os quais fazem parte das estratégias para prevenir possíveis incapacidades, reduzindo o estigma da doença e a exclusão social, além de abordar de maneira sistemática e qualificada o desenvolvimento de ações de vigilância (Gama et al., 2020; Leite et al., 2020).

Ademais, sabe-se que a Atenção Básica detém ferramentas vantajosas para o manejo adequado da hanseníase, haja vista que esse nível tem como alicerce os princípios da equidade e da acessibilidade, apesar dessas ferramentas necessitarem de um aprimoramento, tanto por gestores, quanto por profissionais de saúde, no quesito de se obter maior resolutividade para casos de hanseníase e, em última instância, melhora no cenário epidemiológico da doença no país. Nesse contexto, parte-se de um processo coletivo de trabalho, atuando diretamente nas ações de controle da hanseníase, seja individualmente com o portador da doença, seja com sua família ou a comunidade (Bordon et al., 2019; Nascimento et al., 2020; Ramos, Costa, \& Santos, 2019).

Portanto, realizar ações educativas em saúde torna-se um fator primordial dentro da rede de cuidados, posto que promovem a modificação dos determinantes sociais ao levar para a população os conhecimentos necessários acerca da prevenção das doenças. A educação em saúde ainda destaca-se por ser diversificada e poder ser realizada de diversas maneiras, aplicável tanto na área da saúde como na educação e, por conseguinte, trazendo a participação do usuário do sistema como ferramenta chave de transformação, subsidiando novas condutas e hábitos em saúde (Coelho et al., 2021; Ribeiro et al., 2017).

Por fim, enfatiza-se que a educação em saúde pode contribuir para a ressignificação da doença, promovendo escolhas e possibilidades para adaptação, bem como a quebra de paradigmas acerca da doença. Logo, nesse cenário, o profissional de saúde, juntamente a equipe do local, irá promover a integralidade da assistência aos indivíduos e seus familiares, sendo responsável pelo empoderamento desses indivíduos e replicação de ações preventivas. Para mais, salienta-se que os acadêmicos adquirem uma tarefa ímpar nessa conjuntura, ao desenvolverem habilidades e competências que a futura profissão exigirá, haja vista que a educação no âmbito da saúde está constantemente presente na assistência à saúde (Coelho et al., 2021; Feitosa et al., 2018; Silva, 2020).

\section{Considerações Finais}

A partir dos resultados encontrados neste estudo, identificou-se a necessidade de se trabalhar a temática da profilaxia da hanseníase no ambiente da atenção básica, uma vez que este é um local propício para atividades de educação em saúde dentro dos serviços da rede de saúde. Assim, identificou-se a partir da aplicação da ação, que foi possível fomentar a sensibilização dos participantes acerca da doença, além de disseminar os conhecimentos a nível comunitário.

Ademais, no que tange a participação do acadêmico de saúde, ratifica-se que sua atuação diretamente na realidade possibilita a obtenção de habilidades e competências que serão fundamentais para sua atuação futura, uma vez que promove a obtenção de um olhar holístico para as diversas demandas da população, além de uma conduta acolhedora e equânime, respeitando as diferentes necessidades dos indivíduos. Para mais, essa participação também promove a troca mútua de conhecimentos e o aprimoramento de práticas assistenciais.

Outrossim, destaca-se neste trabalho que o uso de tecnologias leves promove ganho significativo no contexto do 
repasse de informações, haja vista que ela, juntamente com atividades educativas, promove maior autonomia e participação do usuário, fazendo com que este torne-se o centro da ação e possa trazer suas vivências e suas indagações de maneira facilitada e acessível.

Por fim, espera-se que este trabalho possa fomentar novos estudos sobre o tema, visando a criação de estratégias sólidas para a mitigação desta patologia através de medidas educativas profiláticas, em especial no âmbito da atenção primária. Para estudos futuros sugere-se realizar essas ações com um maior número de participantes e em diferentes unidades de saúde, uma vez que essas medidas promovem maior variabilidade de resultados, além de propiciar maior alcance das atividades educativas em saúde.

\section{Referências}

Amaral, M. C. S., Pontes, A. G. V., \& Silva, J. V. (2014). O ensino de Educação Popular em Saúde para o SUS: experiência de articulação entre graduandos de enfermagem e Agentes Comunitários de Saúde. Interface - Comunicação, Saúde, Educação, 18(Supl. 2), 1547-1558.

Araújo, V. S., Dias, M. D., \& Bustorff, L. A. C. V. (2011). A instrumentalização da educação em saúde na atenção básica. Revista de Enfermagem Referência, $3(5), 7-17$.

Berbel, N. (2011). As metodologias ativas e a promoção da autonomia de estudantes. Semina: Ciências Sociais e Humanas, 32(1), 25-40. http://www.uel.br/revistas/uel/index.php/seminasoc/article/view/10326/0.

Bordon, B. P., Souza, L. S., Guimarães, L. B., Ajalla, M. E. A., \& Pinto, C. D. B. S. (2019). O manejo da hanseníase na Atenção Básica: um relato de caso. Perspectivas Experimentais e Clínicas, Inovações Biomédicas e Educação em Saúde, 5(1), 48-53. http://www.seer.ufms.br/index.php/pecibes/index.

Coelho, L. A. C., Lopes, L. S., Bittencourt, M. C., Pereira, A. J. A., Panzetti, T. N. M., Costa, B. N. T., Negrão, R. J. S., Sales, F. S. R., Costa, T. S., Ferreira, I. P., Braga, S. A. M., Silva, J. M. L., Gomes, V. R., Vieira, J. S., Oliveira, A. L. R., \& Magno, J. V. E. P. (2021). Educação em saúde na prevenção ao câncer de mama em uma Estratégia Saúde da Família em Belém-PA. Research, Society and Development, 10(4), 1-8. http://dx.doi.org/10.33448/rsd-v10i4.13810.

Feitosa, E. M., Sá, M. A. P., Andrade, E. G. S., \& Santos, W. L. (2018). Assistência de enfermagem no rastreamento do câncer de mama. Revista JRG de Estudos Acadêmicos, 1(3). http://revistajrg.com/index.php/jrg/article/view/142/238.

Leite, T. R. C., Silva, I. G. B., Lanza, F. M., Maia, E. R., Lopes, M. S. V., \& Calvacante, E. G. R. (2020). Ações de controle da hanseníase na atenção primária à saúde: uma revisão integrativa. Vittalle - Revista de Ciências da Saúde, 32(3), 175-186.

Meneses, L. S. L., Dias, L. K. B. F., Santos, P. H. S., Neres, M. R. M., Medeiros, R. L., Pimentel, H. F. S., \& Lisboa, J. H. V. (2020). Atuação da enfermagem na prevenção, diagnóstico e tratamento da Hanseníase na atenção primária a saúde em Baião-PA: um relato de experiência. Brazilian Journal of Development, 6(7), 48693-48698. https://dx.doi.org/10.34117/bjdv6n7-495.

Nascimento, C. C. F., Borges, C. V., Gonçalves, A. G. S., Silva, D. N., Sousa, M. S., Soares, T. N., Saldanha, Z. O., \& Santos, S. S. (2020). Incidência Clínica da Hanseníase no Município de Ananindeua, Pará, Brasil, 2014 a 2017. Brazilian Journal of health Review, 3(4), 8409-8419. https:// dx.doi.org/10.34119/bjhrv3n4-094.

Nunes, J. M., Oliveira, E. N., \& Vieira, N. F. C. (2011). Hanseníase: conhecimentos e mudanças na vida das pessoas acometidas. Ciência \& Saúde Coletiva, $16(1), 1311-1318$

Ramos, J. S., Costa, L. M.; \& Santos, W. L. (2019). Dificuldades da enfermagem no manejo da hanseníase na atenção primária. Revista JRG de Estudos Acadêmicos, 2(5).

Ribeiro, M. D. A., Silva, J. C. A., \& Oliveira, S. B. (2018). Estudo epidemiológico da Hanseníase no Brasil: reflexão sobre as metas de eliminação. Revista Panamericana de Salud Pública, 42 (1),1-7.

Ribeiro, W. A., Mariano, E. S., Cirino, H. P., Teixeira, J. M., Martins, L. M., \& Andrade, M. (2017). Educação em saúde aos portadores de hipertensão arterial e diabete mellitus na estratégia saúde da família. Revista Pró-UniverSUS, 8), 110-114. http://editora.universidadedevassouras.edu.br/index.php/RPU/article/view/1002.

Rodrigues, R. N., Arcêncio, R. A., \& Lana, F. C. F. (2021). Epidemiologia da Hanseníase e a descentralização das ações de controle no Brasil. Revista Baiana de Enfermagem, 35(39), 1-12. https; //dx.doi.org/10.18471/rbe.v35.39000.

Gama, R. C. A. D. R., Andrade, B. B., Brito, B. A., Sanches, J. L. T., Inácio, L. R., Gondolo, R. S., Fucuta, V. G., \& Silva, K. D. (2020). Hanseníase na região norte do Brasil: perfil clínico epidemiológico entre 2015 e 2017. Revista de Patologia do Tocantins, 7(3), 47-51. https://dx.doi.org/10.20873/uft.2446$6492.2020 \mathrm{v} 7 \mathrm{n} 3 \mathrm{p} 47$.

Silvestre, M. P. S. A., \& Lima, L. N. G. C. (2016). Hanseníase: considerações sobre o desenvolvimento e contribuição (institucional) de instrumento diagnóstico para vigilância epidemiológica. Revista Pan-Amazônica de Saúde, 7(esp), 93-98.

Silva, D. M., \& Sousa, M. N. A. (2021). Prevalência da hanseníase no Brasil e os desafios da busca ativa na atenção primária em saúde. Revista Científica Integração, 2(1), 1-11. http://integracao.unifip.edu.br/. 
Research, Society and Development, v. 10, n. 7, e26610715702, 2021

(CC BY 4.0) | ISSN 2525-3409 | DOI: http://dx.doi.org/10.33448/rsd-v10i7.15702

Silva, R. (2020). Desafios ao diagnóstico da hanseníase na estratégia de saúde da família. Trabalho de conclusão de curso - bacharelado em Enfermagem, Faculdade de Educação e Meio Ambiente - FAEMA.

Sousa, G. S., Silva, R. L. F., \& Xavier, M. B. (2017). Hanseníase e Atenção Primária à Saúde: uma avaliação de estrutura do programa. Saúde em Debate, 41(112), 230-242.

Sousa, S. M., Bernardino, E., Crozeta, K., Peres, A. M., \& Lacerda, M. R. (2017). Cuidado integral: desafio na atuação do enfermeiro. Revista Brasileira de Enfermagem, 70(3), 504-510. 